Cesar V. Villafuerte Jr., MD, MHA ${ }^{1,2}$

Alexander Edward S. Dy, MD²

José Florencio F. Lapeña Jr., MA, MD1,2

'Department of Otorhinolaryngology College of Medicine - Philippine General Hospital

University of the Philippines Manila

${ }^{2}$ Department of Otorhinolaryngology

Philippine General Hospital

University of the Philippines Manila
Correspondence: Dr. Cesar V. Villafuerte Jr.

Department of Otorhinolaryngology

Philippine General Hospital Ward 10

University of the Philippines Manila

Taft Avenue, Ermita, Manila 1000

Philippines

Telephone: (632) 554 8467; (632) 5548400 local 2152

Email address: cvillafuertemd@gmail.com

The authors declared that this represents original material that is not being considered for publication or has not been published or accepted for publication elsewhere in full or in part, in print or electronic media; that the manuscript has been read and approved by the authors, that the requirements for authorship have been met by the authors, and that the authors believe that the manuscript represents honest work.

Disclosures: The authors signed disclosures that there are no financial or other (including personal) relationships, intellectual passion, political or religious beliefs, and institutional affiliations that might lead to a conflict of interest.

\section{An Initial Survey of Septorhinoplasty in Crooked Nose Deformities}

\begin{abstract}
Objective: Crooked nose deformity is a commonly seen reason for septorhinoplasty in the otolaryngology clinic. The purpose of this study is to initially determine the different etiologies of patients with crooked nose deformities who underwent septorhinoplasty, and to describe the different types of crooked nose by their level of deviation and surgical management in our institution.
\end{abstract}

Methods:

Design: Case Series

Setting: $\quad$ Tertiary Public University Hospital

Participants: A chart review of all patients with a crooked nose deformity who were admitted at the otorhinolaryngology ward of the National University Hospital and underwent septorhinoplasty from January 2012 to January 2015 was conducted, and data consisting of age, sex, etiology of crooked nose deformity, level of deviation, cartilage source, and surgical intervention were obtained and analyzed.

Results: A total of 21 patients underwent septorhinoplasty for crooked nose deformity in the study period. The most common etiology for crooked nose was physical violence (13/21 or $62 \%)$, followed by sports injury (4/21 or $19 \%)$, vehicular accidents ( $2 / 21$ or $9 \%)$, and accidental fall (1/21 or $5 \%)$. There were more upper and middle third deviations than lower third deviations. Sixteen out of 21 patients (76\%) underwent open rhinoplasty, while the rest underwent an endonasal approach. Twelve (57\%) underwent intervention on the nasal fracture after at least a year (old or neglected fracture) as compared to the 9 (43\%) who had immediate intervention after less than two weeks. Thirteen used septal cartilage, while 4 used conchal cartilage, and 1 used tragal cartilage. The most common grafts used were spreader and camouflage, followed closely by dorsal onlay, and columellar strut grafts.

Conclusions: The majority of crooked nose deformities that were subjected to septorhinoplasty in our department were secondary to old nasal bone fractures caused by physical violence. Upper and middle third level deviations were more common, and most underwent open rhinoplasty with autologous cartilage grafts. Future studies may increase our understanding of, and improve our techniques in septorhinoplasty for crooked nose deformities in Filipino noses in particular, and Asian noses in general.

Keywords: rhinoplasty, deviated nose, crooked nose, septorhinoplasty, nasal trauma

Crooked nose deformity, also known as deviated nose, twisted nose, asymmetric nose, and scoliotic nose ${ }^{1}$ is a common reason for otorhinolaryngology clinic consult and emergency room referral, with patients usually complaining of nasal stuffiness, nasal obstruction, or an obvious aesthetic deformity, such as a depressed nasal dorsal profile. ${ }^{2}$ Often managed with septorhinoplasty, the etiologic causes of crooked nose are varied, ranging from congenital causes to post-operative complications. These have also changed over the years: infectious and toxic causes have become less frequent, while trauma and primary or secondary reduction 


\section{ORIGINAL ARTICLES}

rhinoplasties now represent the main causes of these deformities. ${ }^{3}$ An extensive literature search of HERDIN NeON, MEDLINE, and Google Scholar using the terms "crooked nose," "nasal bone fracture," "septorhinoplasty" yielded no prior local study on the prevalence of the various etiologies and profiles of crooked nose deformity patients undergoing septorhinoplasty. This lack of information hinders us from proposing guidelines to prevent this condition. Thus, this study aims to initially describe the different etiologies of crooked nose, the different types of crooked nose by their level of deviation, and the different management techniques used on crooked nose deformities among patients undergoing septorhinoplasty in a tertiary public university hospital in the Philippines.

\section{METHODS}

With institutional review board approval, records of all patients who were admitted to the public (charity) ward of the otorhinolaryngology department of the Philippine General Hospital and who had undergone any intervention for crooked nose deformity from January 2012 to January 2015 were retrieved and considered for inclusion in the series. Patients who underwent septorhinoplasty were included, while those with nasal bone fractures who underwent closed reduction under local anesthesia at the emergency room and outpatient department were excluded. Patients who refused any surgical intervention for the deformity were likewise excluded.

Age, sex, etiology of crooked nose deformity, level of deviation, cartilage source, type of surgical intervention, and onset of deformity (acute versus chronic) were recorded.

The level of deviation was divided into: upper third, defined as involvement of the nasal bone and the nasal process of the upper maxillary bone; middle third, defined as involvement of the cartilaginous septum and upper lateral cartilages; and lower third, defined as involvement of the caudal septum and lower lateral cartilages. ${ }^{4}$

Deformities were classified as acute if they were incurred 2 weeks or less before the contemplated intervention, and chronic if they were incurred more than 2 weeks before the contemplated intervention.

Data were tabulated using Microsoft Excel Version version 16 (Microsoft Corporation, Redmond, WA, USA) and examined for potential trends. Strict confidentiality was observed, with all data encoded into electronic abstraction sheets. One sheet (the correlation tool) contained the patient name, medical record number, and patient study number. The patient study numbers were used in all subsequent data tables. The protocol was reviewed and approved by the institutional ethics review board <UPMREB 2014-442-01>

\section{RESULTS}

A total of 21 patients who underwent septorhinoplasty for crooked nose deformity were included in the study. Seventeen (81\%) were male and 4 (19\%) were female. Ages ranged from 13 to 48 years, with the mean age being 26 years. Nasal trauma was found to be the most common cause of crooked nose deformity in the population (20 out of 21 or $95 \%)$. Nasal trauma occurred as a result of physical violence (13 cases), sports injuries (4 cases), vehicular accidents (2 cases), and accidental fall (1 case). Among the cases associated with physical violence, 1 patient presented with both a cleft lip-nose deformity and a nasal bone fracture. One other patient was admitted for revision septorhinoplasty due to a crooked nose deformity from a previous rhinoplasty.

Classifying deformities according to level of deviation yielded 10 cases (48\%) of upper-third deviations, 8 cases (38\%) of middle-third deviations, 1 case (5\%) of combined upper- and middle-third deviation, and 2 cases (10\%) of combined middle- and lower-third deviations. None of the cases exclusively involved the lower-third level.

Eight of the cases (38\%) were acute injuries, while $13(62 \%)$ were chronic. Of the latter, 1 had persistence of the deformity despite initial surgery.

All patients underwent surgery. Open rhinoplasty was the more common approach (16 cases or $75 \%$ ), and this was used in all patients who required autologous cartilage grafting (for spreader grafts, camouflage or augmentation). Only 5 of the surgeries (24\%) employed an endonasal approach. All patients with upper-third deviations underwent lateral and medial osteotomies. None of the cases required intermediate osteotomy. All lateral osteotomies were done with infractures of the nasal bones.

Among the 16 patients who underwent open rhinoplasty, 10 (63\%) utilized autologous cartilage grafts. Of these, 3 had pure camouflage grafts, 2 had combined spreader and columellar strut grafts, 2 had combined spreader and dorsal onlay grafts, 1 had combined spreader and camouflage grafts, 1 had combined camouflage and dorsal onlay grafts, and 1 had combined spreader, camouflage and alar batten grafts. Six patients did not have any cartilage grafts.

The sources of cartilage grafts were as follows: septal cartilage ( 5 cases or $50 \%$ ), conchal cartilage ( 3 cases or $30 \%$ ), tragal cartilage ( 1 case or $10 \%)$, and combined septal and conchal cartilages (1 case or $10 \%)$. The results are summarized in Table 1.

\section{DISCUSSION}

The crooked nose is both a cosmetic and a functional problem, usually affecting nasal breathing. In fact, a twisted nose frequently results in a compromised airway. ${ }^{5}$ In our patients, 15 (71\%) underwent septoplasty, which was done to correct the deviated septum and improve nasal airway. All patients underwent rhinoplasty, as studies show that those who undergo only closed reduction are usually not content. Also, the deviation is usually inadequately reduced, warranting another surgery. ${ }^{6}$

A majority of our patients with crooked nose deformity were males with a mean age of 26 years. This may be related to the finding that the most common etiology of this deformity was trauma, most frequently due to physical violence, followed by sports related injuries, both of which more commonly involve males. ${ }^{7}$ These data are consistent with results of previous demographic and epidemiologic studies on the Asian nose. ${ }^{6}$

One of the patients had acquired the deformity from a previous rhinoplasty. Removal of the dorsal nasal hump with a deviated septum 
ORIGINAL ARTICLES

Table 1. Summary of results

\begin{tabular}{|c|c|c|c|c|c|c|c|}
\hline AGE & SEX & ETIOLOGY & $\begin{array}{l}\text { LEVEL OF } \\
\text { DEVIATION }\end{array}$ & TIME & APPROACH & GRAFTS & $\begin{array}{c}\text { SOURCE OF } \\
\text { CARTILAGE } \\
\text { GRAFT }\end{array}$ \\
\hline 36 & $\mathrm{~F}$ & Vehicular accident & Middle & Chronic & Open & Spreader, camouflage & Septal \\
\hline 48 & $\mathrm{~F}$ & Revision & Middle and lower & Chronic & Open & None & Not applicable \\
\hline 19 & M & Physical violence & Upper and middle & Chronic & Open & $\begin{array}{l}\text { Spreader, alar batten, } \\
\text { camouflage }\end{array}$ & Septal \\
\hline 26 & M & Accidental fall & Upper & Acute & Endonasal & None & Not applicable \\
\hline 13 & $M$ & Sports injury & Middle & Chronic & Open & None & Not applicable \\
\hline 21 & $F$ & Vehicular accident & Upper & Acute & Endonasal & None & Not applicable \\
\hline 45 & $M$ & Sports injury & Upper & Chronic & Open & Camouflage & Conchal \\
\hline 24 & M & Physical violence & Middle & Chronic & Open & Camouflage & Conchal \\
\hline 27 & M & Physical violence & Middle & Chronic & Open & None & Not applicable \\
\hline 20 & $M$ & Sports injury & Upper & Chronic & Open & None & Not applicable \\
\hline 20 & $M$ & Physical violence & Upper & Acute & Endonasal & None & Not applicable \\
\hline 20 & M & Physical violence & Middle & Acute & Endonasal & None & Not applicable \\
\hline 19 & $M$ & Sports injury & Upper & Chronic & Open & Camouflage, dorsal onlay & Septal \\
\hline 18 & M & Physical violence & Upper & Acute & Open & Columellar strut, spreader & Septal and conchal \\
\hline 37 & $\mathrm{~F}$ & Physical violence & Lower and middle & Chronic & Open & Camouflage & Tragal \\
\hline 33 & M & Physical violence & Middle & Acute & Open & Spreader, dorsal onlay & Septal \\
\hline 24 & $M$ & Physical violence & Middle & Acute & Endonasal & None & Not applicable \\
\hline 22 & M & Physical violence & Upper & Chronic & Open & None & Not applicable \\
\hline 36 & $M$ & Physical violence & Upper & Chronic & Open & Spreader, dorsal onlay & Conchal \\
\hline 28 & M & Physical violence & Upper & Chronic & Open & None & Not applicable \\
\hline 17 & $M$ & Physical violence & Middle & Acute & Open & Columellar strut, spreader & Septal \\
\hline
\end{tabular}

or a contraction from healing is the usual cause of deformity in postrhinoplasty patients ${ }^{8}$ and may also have been true in this patient.

About half of the patients had upper third deformities, involving the paired nasal bones. All of them underwent open reduction with in-fracture osteotomies of the nasal bones on lateral osteotomy, and septoplasty. External nasal trauma causes damage to both septal cartilage and nasal bone that cannot be adequately restored by closed reduction. ${ }^{9}$ In these patients, medial and lateral osteotomies would have allowed correction of the nasal bone defects, and septoplasty could have corrected any nasal septum deflections. Two of the 10 patients needed spreader grafts. Spreader grafts both straighten the septal dorsum, and increase the angle at the middle vault to improve the airway. ${ }^{5}$ Another 2 of the 10 patients were given camouflage grafts. This type of graft creates the illusion of a midline position or straight configuration by filling in depressions with thin cartilage. ${ }^{10}$

Eight patients (38\%) had middle third deformities. All of them underwent septoplasty as fractures at this level usually affect the cartilaginous septum. Spreader grafts were utilized in 3 of the 8 patients. One had an additional dorsal onlay graft and another had a columellar strut graft. Dorsal onlay grafts were used to establish nasal contour while columellar strut grafts were used to establish tip support.

A few patients had nasal deformity involving more than one level. A patient who had an existing cleft lip-nose deformity had a nasal bone fracture, with the crooked nose deformity involving both upper and middle third deviations. He underwent a combined cleft rhinoplasty and septorhinoplasty using spreader grafts, alar batten grafts, and camouflage grafts. Alar batten grafts add support to a weakened or pinched nasal valve. Together with the spreader graft, the camouflage graft, and the alar batten graft, the tip plasty done corrected the collapsed nostril and nasal ala present in the cleft lip-nose deformity.

Two patients had both a lower third level deviation and a middle level deviation. This includes the patient whose deformity was from a previous rhinoplasty. No patient had an isolated lower level deviation probably because this level is mostly occupied by cartilage that is more resilient to damage or injury. Both patients underwent open rhinoplasty, which offers the advantage of better field exposure, and better anatomical detail. However, not all combined level deviations warrant exposure. 


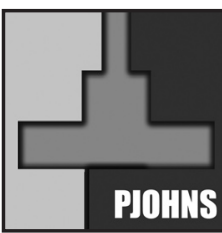

Four of our patients who desired augmentation of the dorsum were given an onlay septal cartilage graft. The Filipino nose (like other Southeast Asian noses) is characterized by a depressed nasal dorsum, and augmentation rhinoplasty is needed for patients who desire a higher nasal dorsum.

Although the debate about open rhinoplasty versus endonasal rhinoplasty is still present, the rhinoplasty surgeon must be adept in both approaches. ${ }^{11}$ However, proficiency at open rhinoplasty is a prerequisite to performing endonasal rhinoplasty, and the latter requires a steeper learning curve. Being in a teaching hospital, the majority of our cases employed an open rhinoplasty approach. Only those with pure upper third bony deviations underwent endonasal septorhinoplasty. For lower third deformities, open rhinoplasty would be preferable for adequate surgical exposure, but an endonasal approach could also be performed. The advantages of utilizing an open approach are the ease of diagnosing structural problems by direct visualization, and simpler technique. It is thought to result in a more predictable outcome resulting from the surgeon's ability to directly and systematically address each part of the nasal framework. The drawbacks include added length of procedure, nasal scars, prolonged edema, and need for more structural grafts as opening the nose results in loss of some support. A closed rhinoplasty allows more expeditious postoperative recovery and, if properly executed, produces the desired results.

Twelve (57\%) patients underwent a form of intervention for their nasal fractures after at least a year, while $9(43 \%)$ had intervention done in less than two weeks. Thus, a majority of the cases in our population were classified as old or neglected nasal bone fractures. These deformities are harder to correct since malunion has already happened. Most cases of neglected fractures are due to economic problems where the family had to prioritize more urgent needs.

We utilized septal, conchal and tragal cartilages as graft donor sites in our series. Septal grafts were used for spreader and columellar struts as well as dorsal onlay grafts. Conchal cartilage was used for camouflage and dorsal augmentation grafts, although a double layer conchal cartilage graft can also be used as a columellar strut. ${ }^{12}$ Where smaller cartilage grafts were needed (such as for camouflage), we utilized tragal cartilage which is ideal if no longer than $20 \mathrm{~mm}$ is required. ${ }^{13}$

In our initial series, the majority of patients who underwent septorhinoplasty for deformity had crooked noses secondary to old nasal bone fractures, and the open rhinoplasty approach was used more often. Patients were managed based on the level of deformity (upper, middle or lower third or mixed) with most undergoing septorhinoplasty, septoplasty, contour and/or camouflage grafting. All grafts used were autologous (septum, conchal or tragal cartilage) and were used as different types of grafts (spreader, alar batten, dorsal onlay and others); no silicone or EPTFE implants were used in any of the cases. We did not have any postoperative problems with the cartilage grafts, as we did not use rib cartilage in any of our cases. Rib cartilage grafts have been reported to have warping as a major complication.

A major limitation in this initial survey was inadequate documentation and follow-up, to substantiate and compare our interventions and outcomes. This series has other limitations. First, we only described patients with crooked nose deformities who underwent septorhinoplasty, and not all patients with crooked nose deformity. This sampling bias prevents us from applying our findings to crooked nose deformities in general. Second, we described levels of deformity and corresponding management, but not the reasons for choosing these approaches, nor the outcomes of these types of management. For instance, it would have been helpful to evaluate the post-operative effects of osteotomy and septoplasty on nasal bone defects and septal deflections; spreader graft effects on nasal airway; camouflage graft effects on aesthetics; dorsal onlay and columellar strut grafts on nasal contour and tip support; and alar batten grafts on the nasal valve instead of merely enumerating these interventions per level of deformity. In our lateral osteotomies we followed the high-low-high lateral osteotomy method starting at the upper part of the inferior turbinate attachment. ${ }^{1}$ This was to avoid in-fracture movement of the inferior turbinate which could contribute to post-operative nasal obstruction. Moreover, a comparison of open and closed rhinoplasty outcomes for similar deformity levels or of similar procedures among fresh and neglected fractures would be helpful, and future studies should consider these. Our documentation of pre-operative symptoms and deformities and post-operative outcomes should be improved in order to facilitate such studies, but we have yet to find a solution to poor follow-up by patients who are presumably happy with their outcomes, or who simply cannot afford repeated and prolonged consultations. Finally, we were not able to show before and after pictures, due to lack of consent to publish photographs in full.

Future studies addressing these limitations may increase our understanding of, and improve our techniques in septorhinoplasty for crooked nose deformities in Filipino noses in particular, and Asian noses in general.

\section{REFERENCES}

1. Jang YJ. Practical Septorhinoplasty - An Asian Perspective. $1^{\text {st }}$ Ed, Seoul: Koon Ja Publishing Inc; 2007; p210.

2. Boccieri A. The crooked nose. Acta Otorhinolaryngol Ital. 2013 Jun; 33(3):163-168.

3. Durbec M, Disant F. Saddle nose: classification and therapeutic management. Eur Ann Otorhinolaryngol Head Neck Dis. 2014 Apr; 131(2): 99-106.

4. Hussein WKA. Crooked nose: An update of management strategies. EJENTAS. 2015 Mar;16(1):1-7.

5. Hoffman, J. Management of the twisted nose. Oper Tech Otolaryngol Head Neck Surg, 1999 Sept; 10(3):232-237.

6. Lee $\mathrm{DH}$, Jang YJ. Pediatric nasal bone fractures: Does delayed treatment really lead to adverse outcomes? Int J Pediatr Otorhinolaryngol. 2013 Jan; 77(5): 726-731.

7. Liu C, Legocki AT, Mader NS, Scott AR. Nasal fractures in children and adolescents: Mechanisms of injury and efficacy of closed reduction. Int J Pediatr Otorhinolaryngol. 2015 Dec. 10(3):232237.

8. Behrbohm H, Tardy ME. Essentials of septorhinoplasty. Thieme Stuttgart-New York, 2004: 162

9. Haack S, Gubisch W. Reconstruction of the septum with an autogenous double-layered conchal L-strut. Aesthetic Plast Surg. 2014 Oct; 38(5):912-922.

10. Ducic Y, Defatta R. Closed rhinoplasty. Operative techniques in otolaryngology. 2007 Sept; 18(3): 233-242.

11. Teymoortash A, Fasunla JA, Sazgar AA. The value of spreader grafts in rhinoplasty: a critical review. Eur Arch Otorhinolaryngol. 2012 May; 269(5):1411-1416.

12. Zinser MJ, Siessegger M, Thamm O, Theodorou P, Maegele M, Ritter L, et al. Comparison of different autografts for aural cartilage in aesthetic rhinoplasty: is the tragal cartilage graft a viable alternative? Br J Oral and Maxillof Surg. 2013 Dec;51(8):863-867.

13. Scattolin A, D'Ascanio L. Grafts in "closed" rhinoplasty. Acta Otorhinolaryngol Ital. 2013 Jan;33(3):169-176. 